\title{
Increased Cellularity of Tumor-Encased Native Vessels in Prostate Carcinoma Is a Marker for Tumor Progression
}

Fernando U. Garcia, M.D., Cullen A. Taylor, M.D., J. Steve Hou, M.D., Daniel B. Rukstalis, M.D., Mark E. Stearns, Ph.D.

Departments of Pathology (FUG, CAT, JSH, MES) and Urology (DBR), MCP-Hahnemann University, Philadelphia, Pennsylvania

Changes in the native vasculature of the prostate gland associated with prostate adenocarcinoma have not been well characterized. Eighty-nine whole mounts of entirely submitted radical prostatectomies were reviewed. Thirty prostates with a minimum of five native arteries surrounded by carcinoma with corresponding control arteries were found and included in this study. The number of nuclei in the media of native arteries was recorded per $0.138 \mathrm{~mm}^{2}$ using a $40 \times$ objective.

The number of nuclei in vessels embedded in carcinoma $(n=204)$ was increased when compared with controls (26.37 versus 20.58 mean nuclei per $\left.0.138 \mathrm{~mm}^{2} ; P<.001\right)$. Pathologic Stage T3 carcinomas contained vessels that were more cellular than stage T2 $(P<.001)$. Vessels embedded in Gleason Grade 4 showed more cellularity than arteries embedded in Gleason Grade $3(P<.002)$.

Increased media cellularity of native prostate vessels encased in carcinoma is a histologic feature of higher grade/stage prostate carcinoma and provides positive indicator of advanced prostate cancer.

KEY WORDS: Carcinoma, Histopathology, Prostate, Vasculature.

Mod Pathol 2000;13(7):717-722

The incidence and mortality of prostate adenocarcinoma has been increasing over the past decade. Also, there has been increased awareness of the disease in the general population, which has resulted in early detection of this neoplasm $(1,2)$. In this regard, increased screening with more sensitive

Copyright () 2000 by The United States and Canadian Academy of Pathology, Inc.

VOL 13, NO 7, P. 717, 2000 Printed in the U.S.A.

Date of acceptance: January 27, 2000.

Address reprint requests to: Fernando U. Garcia, M.D., Department of Pathology and Laboratory Medicine, MCP-Hahnemann University, MS 435, 15th and Vine Streets, Philadelphia, PA 19102-1192; e-mail: Fernando.Garcia@drexel.edu; fax: 215-246-5493. diagnostic tests has resulted in early detection of a great number of small carcinomas with unknown malignant potential in biopsy specimens.

The improvements in early detection have increased the demand for pathologists to categorize the biologic behavior of prostate carcinoma. A number of prognostic markers have been suggested to correlate with pathologic stage, including Gleason grade (3); tumor volume (4); DNA ploidy; and expression of various growth factors receptors, oncogenes, and tumor suppressor genes (5). However, more accurate predictors of tumor progression are needed to better advise patients for treatment options.

Recently, the potential correlation of tumor neovascularity with progression of disease has been the focus of considerable study. Tumor neovascularity has been reported to correlate with pathologic stage and overall disease-free survival in bladder carcinoma (6), cutaneous melanoma (7), and prostatic adenocarcinoma (8). It is thought that tumorassociated angiogenic factors (i.e., vascular endothelial growth factors, vascular permeability factor) (9) not only promote neoangiogenesis within the tumor but also induce vascularization of stroma surrounding the tumor. Little or no information is available on the changes in the native vessels of slow-growing human prostate tumors. In this article, we investigate changes in the native vasculature of the prostate gland and correlate these changes with Gleason grade and pathologic stage.

\section{MATERIALS AND METHODS}

\section{Patient Materials}

Radical prostatectomies from 89 patients with a diagnosis of prostatic adenocarcinoma were retrieved from archived materials (Department of Pathology of MCP-Hahnemann University). Thirty prostates with a minimum of five native arteries surrounded by carcinoma with corresponding con- 
trol arteries were found and included in this study. Five arteries were used as a cut point to ensure sufficient data from individual patients. The median age of the patients was 61 years (range, 46 to 77 years). Sagittal whole mount sections from each specimen were cut and submitted for processing after fixation in $10 \%$ neutral buffered formalin as previously described (10). Sections were cut from each block and stained with hematoxylin and eosin. Each case was independently reviewed by at least two pathologists, who determined the diagnosis, histologic grade, and pathologic stage. The pathologic staging of the tumor was done according to American Joint Committee on Cancer/Union Internationale Contre le Cancer staging scheme.

\section{Measurement of Native Vasculature}

The specimens included in the study exhibited a minimum of five native arteries surrounded by tumor and five native control arteries at least two fields of view distance from the tumor foci $(10 \times$ magnification). On average, the arteries were considered native if they were $\geq 0.46 \mathrm{~mm}^{2}$, based on measurements of the outside diameters. This cut point was chosen to separate native vessels from tumor neovasculature and to allow the plot to be placed on the vessel wall. Both study and control arteries were located in the peripheral zone of the prostate.

Nuclei present in the arterial walls were counted using an Olympus BH-2 microscope with a camera lucida (1.25× Olympus, Japan). A $0.138-\mathrm{mm}^{2}$ plot was placed on the most cellular area of each vessel wall using a $40 \times$ objective, and the number of nuclei was recorded. Subsequently, the vessels were grouped according to pathologic stage of the patient and Gleason grade (pattern) of the tumor surrounding each vessel.

\section{Calculation of Tumor Volume}

The tumor volume was calculated using the following formula:

\section{Tumor volume $\left(\mathrm{cm}^{3}\right)$ \\ $=$ percentage of prostate involved by carcinoma} $\times$ prostate volume.

The prostate volume was calculate using the gross measurements of height, width, and length using the formula for calculating volume of an ellipsoid:

$$
V=0.52 \times \text { height } \times \text { length } \times \text { width }
$$

The percentage of prostate volume involved with tumor was calculated using a modification of the grid method (11). Briefly, the areas of tumor were outlined with a marker on the glass slide, and a transparent grid with 5-mm squares was overlaid on the $7.5 \times 5-\mathrm{cm}$ glass slide. The number of squares that the tumor intersected was recorded as well as the number of squares that overlaid the prostatic tissue. A ratio of the total number of squares intersected by carcinoma over the total number of squares over prostatic tissue was calculated for each case, and the percentage of prostate involved by carcinoma was calculated.

\section{Immunohistochemistry}

Sections from formalin-fixed, paraffin-embedded tissue were labeled with smooth muscle actin monoclonal antibody 1A4 (Sigma, St. Louis, MO) for $3 \mathrm{~h}$ after antigen retrieval with citrate buffer $(\mathrm{pH}$ 7.0) and steam for 20 min according to a method previously described (12). The sections were then sequentially incubated with biotinylated immunoglobulin secondary antibody, avidin conjugated horseradish peroxidase, and diaminobenzidine chromogen (Sigma) followed by copper sulfate enhancement and counterstained with hematoxylin. Positive control slides of myometrium were processed with each batch. Negative control slides were also processed using a nonspecific immunoglobulin IgG (Sigma) at the same concentration as the primary antibody. The control slides were processed with the automated staining procedure identical to that used for the primary antibodies.

\section{Statistical Analysis}

Mean differences between tumor and control vessels were compared using a paired $t$ test. Comparisons between patient groups were assessed with unpaired $t$ tests and/or Mann-Whitney $U$ tests. A discriminant analysis was performed to determine whether pathologic stage could be predicted by the cellularity of the vessels encased in carcinoma. A Pearson correlation to asses the relationship between tumor volume and media cellularity was performed. SPSS statistical package, version 6.2 (SPSS, Inc., Chicago, IL) was used for all calculations.

\section{RESULTS}

\section{Changes of Native Arteries Surrounded by Tumor}

In sagittal whole mount sections, the vasculature runs from the peripheral zone at the posterolateral area toward the urethra. The larger arteries are usually located in the peripheral zone near the capsule of the prostate in the posterior lateral area. At least five thickened hypercellular small arteries or larger arterioles $\left(\geq 0.46 \mathrm{~mm}^{2}\right)$ were identified within the tumor (entirely surrounded by tumor) or in the area directly adjacent to the tumor (at least one side of 


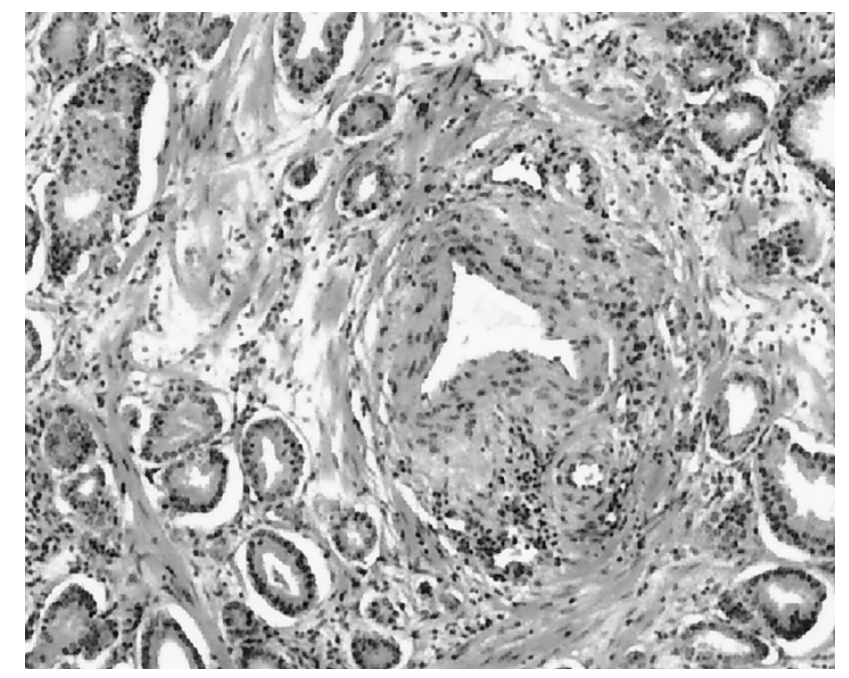

FIGURE 1. Increased media cellularity of native vessels associated with Gleason Grade 3 prostatic adenocarcinoma (hematoxylin and eosin stain; original magnification, $40 \times$ ).

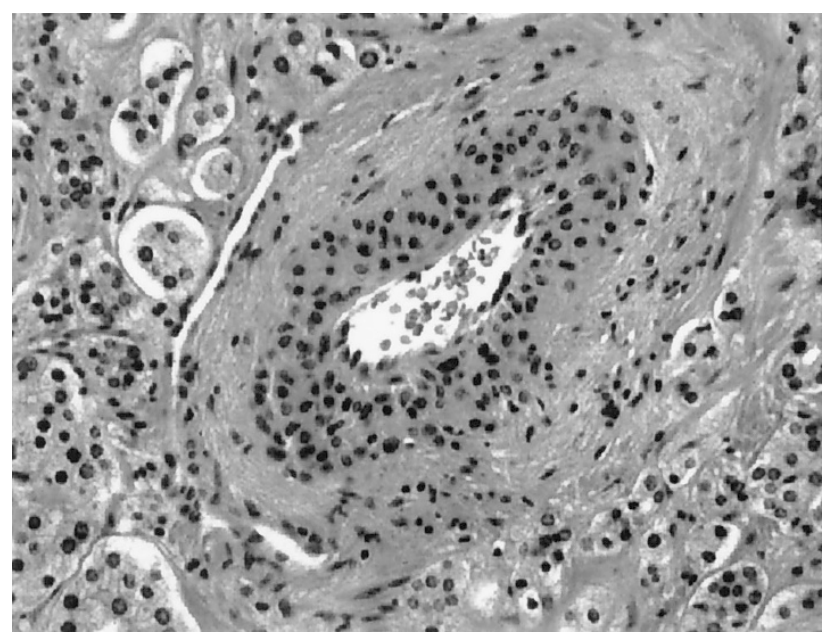

FIGURE 2. Increased media cellularity of native vessels associated with Gleason Grade 4 prostatic adenocarcinoma (hematoxylin and eosin stain; original magnification, $100 \times$ ).

the vessel wall in contact with tumor) in 30 of 89 (34\%) cases studied (Figs. 1 and 2). All vessels studied were located within the prostate and in the peripheral zone. Light microscopic examination of the vessels surrounded by tumor and that of control vessels revealed few differences in the appearance of the endothelial cells. The major differences were found in the media. Vessels in the vicinity of carcinoma had media hypercellularity. The cells had rounder nuclei with abundant eosinophilic cytoplasm, indistinguishable cell borders, and inconspicuous nucleoli arranged in a concentric or haphazard pattern resembling glomus cells (Fig. 3). These cells showed smooth muscle differentiation being positive for smooth muscle actin (Fig. 4). The outer adventitial layer appeared thickened with fibrotic connective tissue, which exhibited a variable degree of hyalinization when compared with con- trol vessels. However, increased cellularity was not present in the adventitia. All of these changes resulted in an increase in thickness of the vessel wall when compared with controls (data not shown). No fibrinoid necrosis of the media, lymphocytes, giant cells, granulomata, or atheromatous changes was observed.

\section{Measurement of Media Cellularity}

Vessels embedded in carcinoma and paired control vessels from each case were identified. A total of 204 native vessels with matched controls were selected from 30 of 89 cases. Media cellularity was counted without knowledge of the pathologic stage and the Gleason grade of the overall tumor nodule. Tumor and control vessels were compared on the per-patient analyses by paired $t$ test. This compar-

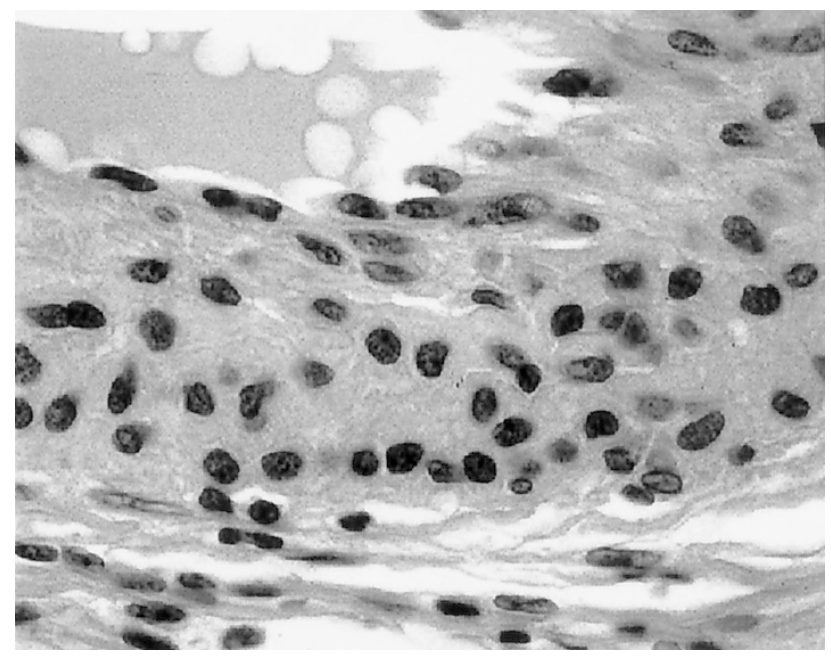

FIGURE 3. The oval-shaped, mildly enlarged, and hyperchromatic smooth muscle cells in the media of a vessel surrounded by adenocarcinoma on higher magnification (hematoxylin and eosin stain; original magnification, $400 \times$ ).

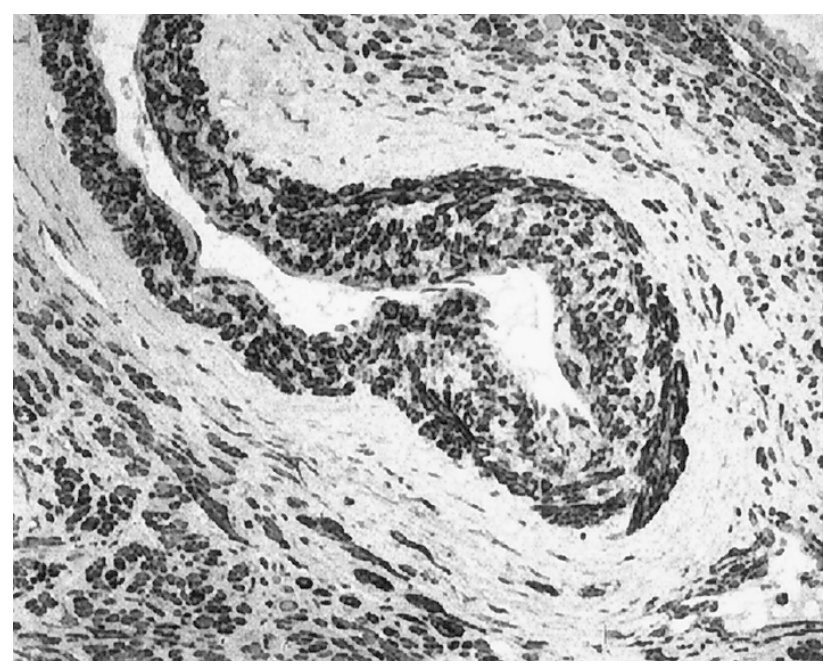

FIGURE 4. Smooth muscle differentiation of cellular changes in the vessel wall (immunoperoxidase stain, smooth muscle actin; original magnification, $100 \times)$. 
ison was significant $(\mathrm{t}=9.7, P<.001)$ with tumor vessels embedded in carcinoma (mean, 26.37; SD, 3.75 ) being more cellular per $0.138 \mathrm{~mm}^{2}$ than control vessels (mean, 20.58; SD, 2.17). The mean difference was 5.78; a 95\% confidence interval indicated that the true mean difference was probably between 4.56 and 7.00. (Fig. 5).

\section{Correlation with pathologic stage}

Vessels from patients with pathologic Stage T2 ( $n=7)$ and stage T3 $(n=23)$ were compared. Vessels embedded in Stage T3 tumors (mean, 27.38; $\mathrm{SD}, 3.54)$ were more cellular than Stage T2 tumors (mean, 23.02; SD, 2.24). The differences were significant, $(P=.005$ by unpaired $t$ test) (Fig. 6). The mean difference was 4.37 in favor of greater cellularity in Stage 3, and a 95\% confidence interval for the true difference was 1.44 to 7.29 . In addition, group-comparison analysis was performed using individual vessels as the unit of analysis. A MannWhitney $U$ test was used because values were not normally distributed. Vessels in pathologic Stage T3 were significantly more cellular than the vessels in Stage T2 disease $(P<.001)$.

To determine whether pathologic Stage T2 versus T3 could be predicted by the cellularity of the vessels encased in carcinoma, discriminant analysis was used. When assuming that both pathologic stages are equally common, the analysis was able to classify 21 of the 30 patients $(70 \%)$ correctly, using the mean number of nuclei within the media of arteries encased in carcinoma for each patient as a predictor. It correctly classified 5 of 7 Stage T2 patients $(71 \%)$ as Stage T2 and 16 of 23 Stage T3 patients $(70 \%)$ as stage T3. If Stage T3 is taken as the "abnormal" and Stage T2 as the "control," then these correspond to a sensitivity of $70 \%$ and a specificity of $71 \%$. Using the same analogy, the positive predictive value of pathologic Stage T3 based on

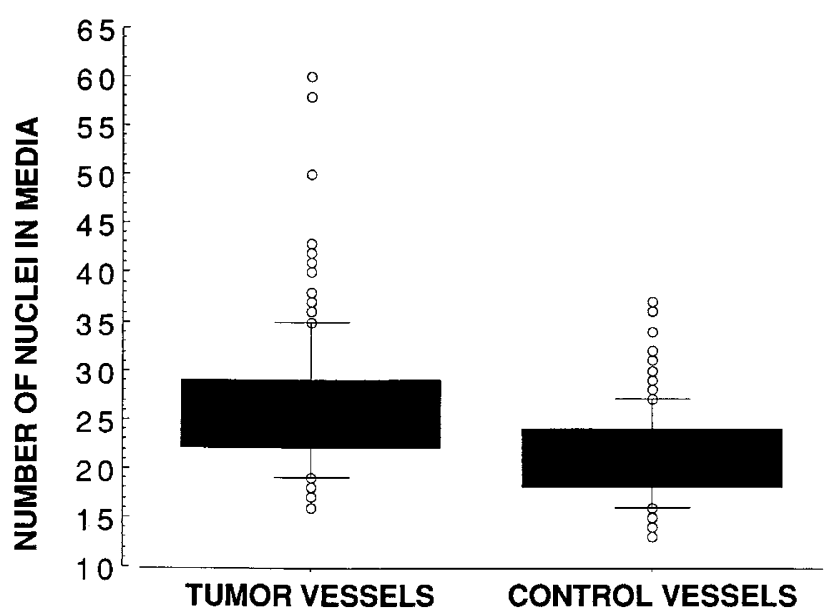

FIGURE 5. Vessels surrounded by carcinoma $(n=204)$ had a higher mean cellularity per $0.138 \mathrm{~mm}^{2}$ than control vessels away from carcinoma; 26.37 (SD, 3.75) versus 20.58 (SD, 2.17) $(P<.001)$.

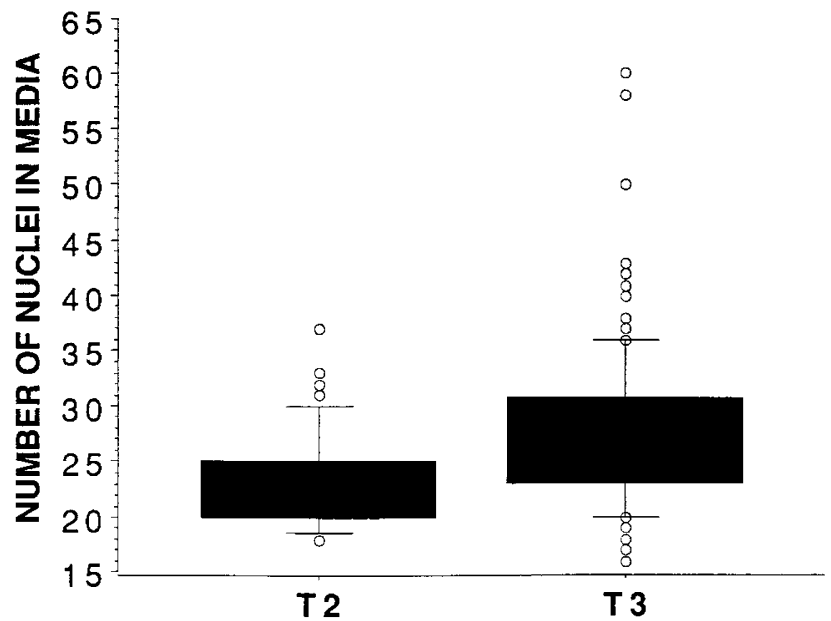

FIGURE 6. The finding of vessels with increased media cellularity correlated with pathologic stage. Vessels from patients with pathologic Stage T2 had a mean cellularity of 23.02 (SD, 2.24), and Stage T3 cases had a mean cellularity of 27.38 (SD, 3.54; $P=.005$ ).

media cellularity would be 16 of 18 (89\%). Unfortunately, the negative predictive value of pathologic Stage T2 is only 5 of $12(42 \%)$.

When using the actual frequency of pathologic stages in a studied population, the analysis classified $87 \%$ correctly. Only 4 of 7 (57\%) Stage T2 patients were correctly classified as pathologic Stage T2 on the basis of media cellularity of vessel encased in carcinoma. However, 22 of 23 (96\%) Stage T3 patients were correctly classified as such. The sensitivity is $96 \%$, with a specificity of $57 \%$. The positive predictive value is 22 of $23=88 \%$, with a negative predictive value of 4 of $5=80 \%$.

\section{Correlation with Gleason grade}

Patients had carcinoma with one or several Gleason grades that encased native vessels. For each patient, a predominant Gleason pattern was selected. In the case of a tie, the highest score was used for the per-patient analyses. Comparing the 18 patients with Gleason pattern 4 (mean, 27.04; SD, 3.86) with the 9 patients with Gleason pattern 3 (mean, 24.17; SD, 2.67), the mean cellularities were significantly different, albeit not so strongly as for pathologic stage (Figs. 1 and 2). The unpaired $t$ test returned a $P$ value of 0.028 , one-tailed. The mean difference was 2.88 in favor of greater cellularity for Gleason 4. The Mann-Whitney $U$ test using the Gleason pattern of the carcinoma surrounding the individual vessels showed significantly more cellularity in Gleason pattern 4 than Gleason pattern 3 $(P=.002)$ (Fig. 7). There were only 12 paired vessels identified in three patients with Gleason pattern 5 . The mean nuclei was $29.50 \pm 0.47$, which was $13.7 \%$ more compared with controls and $6.9 \%$ more than that of the Gleason pattern 4 group. No statistically significant differences were achieved between these 


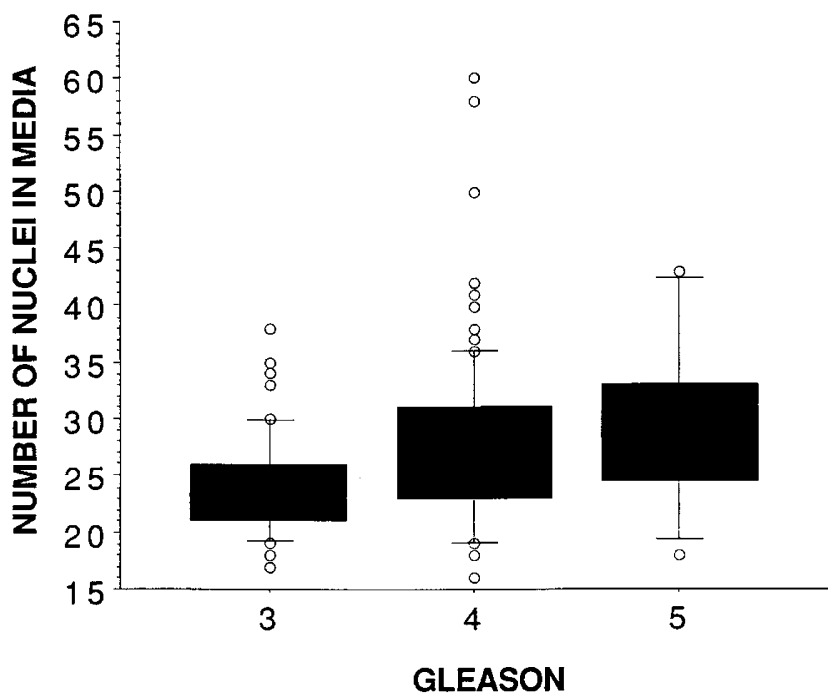

FIGURE 7. The increase media cellularity also correlated with Gleason grade. Vessels embedded in Gleason Grade 3 had a mean cellularity of 24.17 compared with 27.04 for Gleason Grade 4 and 29.5 for Gleason Grade 5 ( $P=.002$ between Gleason 3 and 4 ).

groups. No differences in vessel cellularity were found when vessels were grouped according to size.

\section{Correlation between tumor volume and media cellularity}

The mean tumor volume was $6.91 \pm 1.36 \mathrm{cc}$. Correlation between tumor volume and mean vessel cellularity was analyzed using Pearson correlation. There was no significant correlation between the tumor volume and media cellularity $(r=.34$, $P=.196$ ), although higher volume carcinomas showed a tendency for higher media cellularity.

\section{DISCUSSION}

In this study, we have shown for the first time that within prostatic carcinoma, the walls of native arteries have markedly hypercellular media when compared with controls. In addition, occasional increased fibrosis and focal hyalinization of the adventitial layer was noted in the tumor-associated vessels.

This vascular change had a sensitivity of $96 \%$ of predicting pathologic Stage T3, although it had a low sensitivity of $56 \%$. Because this change is seen in larger vessels that are located mostly in the posterolateral area, it is less likely to be present in biopsy specimens, especially if a mid-lobar approach is used in obtaining the biopsies (13). However, this study demonstrates that when present, it can predict pathologic Stage T3 and therefore should be included in the biopsy report.

Analysis of the relationship of increased media cellularity with pathologic stage showed that increased media cell density correlated closely with higher pathologic stage (Stage T3 versus T2) having a high positive and negative predictive value. The relationship between Gleason grade and increased media cellularity also showed higher media cellularity for Gleason Grade 4 when compared with Gleason Grade 3. Note that although the changes in the Gleason Grade 5 group were not statistically significant, the same trend was apparent.

These findings seem to indicate that arteries with increased media cellularity provide a marker associated with aggressive carcinoma. It is important to point out that by requiring a minimum of 5 arteries ( $>0.46 \mathrm{~mm}^{2}$ in diameter), we inherently selected for carcinomas with a larger tumor volume. This is further demonstrated by having fewer pathologic Stage T2 and Gleason Grade 1 or 2 carcinomas in the study.

There is a need for accurate preoperative predictors of pathologic staging in prostate cancer. Conventional imaging modalities (14), serum prostatespecific antigen levels, Gleason grading, and tumor volume in needle biopsies (15) have been consistently correlated with pathologic findings in prostatectomy specimens in the individual patient (16, 17). However, their predictive value has been inadequate on a patient-by-patient basis, particularly in cases in which moderately differentiated carcinomas (Gleason Grade 3) were diagnosed by needle biopsy (18).

In human tumorigenesis, angiogenic induction plays a critical role in the growth and metastasis of tumors $(19,20)$. Some authors $(8,21,22)$ have established a quantitative relationship between tumor neovascularization with recurrence and progression in prostate cancer, although others have not found correlation with clinical outcome (23). Tumor microvessel density/vascular endothelial growth factor expression has also been shown to predict biologic behavior in prostatic cancer (24, 25). However, no studies have been published on the importance of tumor-associated changes in the native vasculature. We observed that vessels surrounded by prostatic adenocarcinoma have thickened vascular walls, increased media cellularity, and, occasionally, increased fibrosis of the adventitial layer. These changes occurred in approximately one third of cases, are easily seen in the peripheral zone of the prostate, and are significantly different from control vessels in the same patient. More important, these findings seem to be associated with higher pathologic stage and higher Gleason grade, which are indicators of clinically significant prostate carcinoma.

The mechanisms that contribute to increased cellularity of the vessel media are not known. Two possible hypotheses can be put forward. First, this change can be due to a process of recruitment of prostatic stromal cells into the vessel media. In the complex milieu of tumor-stroma interaction, it is 
probable that several factors involved in different aspects of the angiogenic process are required for the increased media cellularity of vessels. Most likely, a group of angiogenic growth factors including angiopoietin-1, platelet-derived growth factor- $\beta$, heparin-binding epidermal growth factor-like growth factor, tissue factor, and endothelin may be released from an interaction between tumor cells and endothelial cells. Release of these angiogenic growth factors into the surrounding stroma would directly stimulate pericytes/smooth muscle cells to migrate into the vessel wall. However, no increased cellularity is seen in the adventitia of the hypercellular vessels (data not shown). We do not favor proliferation because MIB-1 expression in the cells in the media was absent (data not shown). The second hypothesis is related to local increases of intravascular pressure. This can be the result of increased neovascularization within the carcinoma, which in turn could increase the blood flow to areas of carcinoma. With increased blood flow into the carcinoma, localized areas of increased intravascular pressure in the native arteries could be created, resulting in increased cellularity of the media. This phenomenon might be similar to the vascular changes seen in acral arteriovenous tumor where shunting from arterial to venous channels, with subsequent increase of blood flow, give a characteristic thickened and cellular aspect to the media of the vessels (26).

\section{REFERENCES}

1. Krahn MD, Mahoney JE, Eckman MH, Trachtenberg J, Pauker SG, Detsky AS. Screening for prostate cancer. A decision analytic view. JAMA 1994;272:773-80.

2. Albertsen PC, Fryback DG, Storer BE, Kolon TF, Fine J. Longterm survival among men with conservatively treated localized prostate cancer. JAMA 1995;274:626-31.

3. Gleason DF, Mellinger GT. VACURG. Prediction of prognosis for prostatic carcinoma by combined histologic grading and clinical staging. J Urol 1974;111:58-64.

4. Stamey TA, McNeal JE, Freiha FS, Redwine E. Morphometric and clinical studies on 68 consecutive radical prostatectomies. J Urol 1988;139:1235-41.

5. Deitch AD, deVere White RW. DNA flow cytometry and immunohistochemistry of p53 pathway genes as predictive modalities in localized prostate cancer. In: Forster CS, Bostwick DG, editors. Pathology of prostate (MPP 34). 1st ed. Philadelphia: W.B. Saunders; 1998. p. 327-39.

6. Chodak GW, Haudenschild C, Gittes RF, Folkman J. Angiogenic activity as a marker of neoplastic and preneoplastic lesions of the bladder. Annu Surg 1980;192:762-71.

7. Srivastava A, Laidler P, Davies RP, Horgan K, Hughes LE. The prognostic significance of tumor vascularity in intermediatethickness (0.76-0.4 $\mathrm{mm}$ thickness) skin melanoma. Am J Pathol 1988;133:419-23.
8. Brawer MK, Deering RE, Brown M, Preston SD, Bigler SA. Predictors of pathologic stage in prostatic carcinoma: the role of neovascularity. Cancer 1994;73:678-87.

9. Nicosia RF, Villaschi S. Autoregulation of angiogenesis by cells of the vessel wall. Int Rev Cytol 1999;185:1-43.

10. Garcia FU, Shidham VB, Rukstalis DB. Advantages of sagittal sectioning prostatectomies. Mod Pathol 1997;10:76A.

11. Humphrey PA, Vollmer RT. Percentage carcinoma as a measure of prostatic tumor size in radical prostatectomy tissues. Mod Pathol 1997;10:326-33.

12. Lear SC, Janckila AJ, Martin AW, Yam LT. Immunohistochemistry of tartrate-resistant acid phosphatase: assessment of steam heat for epitope enhancement and comparison of B-5 and formalin fixation. J Histotechnol 1997;20:303-6.

13. Hodge KK, McNeal JE, Terris MK, Stamey TA. Random systematic versus directed ultrasound guided transrectal core biopsies of the prostate. J Urol 1989;142:7174-9.

14. Rifkin MD, Zerhouni EA, Gatsonis CA, Quint LE, Paushter DM, Epstein JI. Comparison of magnetic resonance imaging and ultrasonography in staging early prostate cancer: results of a multi-institutional cooperative trial. N Engl J Med 1990; 323:621-6.

15. Ravery V, Schmid H-P, Toublanc M, Boccon-Gibod L. Is the percentage of cancer in biopsy cores predictive of extracapsular disease in T1-T2 prostate carcinoma? Cancer 1996;78: 1079-84.

16. Stamey TA, Yang N, Hay AR, McNeal JE, Freiha FS, Redwine E. Prostate-specific antigen as a serum marker for adenocarcinoma of the prostate. N Engl J Med 1987;317:909-16.

17. Bostwick DG. Gleason grading of prostatic needle biopsies: correlation with grade in 356 matched prostatectomies. Am J Surg Pathol 1994;18:796-803.

18. Di Loreto C, Fitzpatrick B, Underhill S, Kim DH, Dytch HE, Galera-Davidson $\mathrm{H}$, et al. Correlation between visual clues, objective architectural feature and interobserver agreement in prostate cancer. Am J Clin Pathol 1991;96:70-5.

19. Gimbrone MA, Leapman SB, Contran RS, Folkman J. Tumor dormancy in vivo by prevention of neovascularization. J Exp Med 1972;136:261-76.

20. Mahadevan V, Hart IR. Metastasis and angiogenesis. Acta Oncol 1990;29:97-102.

21. Bettencourt MC, Bauer JJ, Sesterhenn IA, Connelly RR. Moul JW. CD34 immunohistochemical assessment of angiogenesis as a prognostic marker for prostate cancer recurrence after radical prostatectomy. J Urol 1998;160:459-65.

22. Silberman MA, Partin AW, Veltri RW, Epstein JI. Tumor angiogenesis correlates with progression after radical prostatectomy but not with pathologic stage in Gleason sum 5 to 7. Cancer 1997;79:772-9.

23. Rubin MA, Buyyounouski M, Bagiella E, Sharir S, Neugut A, Benson M, et al. Microvessel density in prostate cancer: lack of correlation with tumor grade, pathologic stage, and clinical outcome. Urology 1999;53:542-7.

24. Harper ME, Glynne-Jones E, Goddard L, Thurston VJ, Griffiths K. Vascular endothelial growth factor (VEGF) expression in prostatic tumors and its relationship to neuroendocrine cells. Br J Cancer 1996;74:910-6.

25. Jackson MW, Bentel JM, Tilley WD. Vascular endothelial growth factor (VEGF) expression in prostate cancer and benign prostatic hyperplasia. J Urol 1997;157:2323-8.

26. Connelly MG, Winkelmann RK. Acral arteriovenous tumor: a clinicopathologic review. Am J Surg Pathol 1985;9:15-21. 